\title{
Underlying Mechanisms for Developing Process Signatures in Manufacturing
}

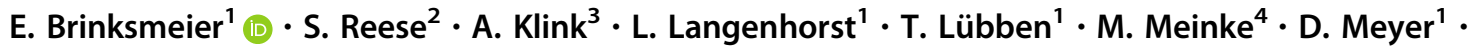 \\ O. Riemer $^{1} \cdot$ J. Sölter ${ }^{1}$
}

Received: 13 February 2018/Revised: 25 July 2018 / Accepted: 7 August 2018/Published online: 3 September 2018

(C) The Author(s) 2018

\begin{abstract}
The concept of Process Signatures allows the reliable and knowledge-based prediction of material modifications (e.g., changes of hardness, residual stress, microstructure and chemical composition) researchers in the field of surface integrity, and manufacturing technologies have already been seeking for a long time. A Process Signature is based on the correlation between the internal material loads in manufacturing processes (e.g., stress, strain, temperature) and the resulting material modifications. The target of this paper is to get a comprehensive view on the development of single Process Signature Components for processes with different predominant impacts. Particularly, a mechanism-based approach leading to significant descriptives of internal material loads for a specific material modification is proposed. By this, a deeper understanding of the underlying mechanisms leading to changes of the workpiece surface layer properties caused by manufacturing processes is provided. The challenging identification of significant mechanisms and descriptives of internal material loads is highlighted for each process, and supporting measurement methods and modeling approaches are presented. Process Signatures are expected to enable the solution of the so-called inverse problem in manufacturing.
\end{abstract}

Keywords Surface integrity $\cdot$ Machining $\cdot$ Microstructure $\cdot$ Process Signatures

\section{List of symbols}

$\mathrm{A}_{\mathrm{c} 1} \quad$ Transformation start temperature $\left({ }^{\circ} \mathrm{C}\right)$

$c \quad$ Phase fraction (\%)

$c_{0} \quad$ Initial phase fraction (\%)

$d_{\mathrm{b}} \quad$ Tool diameter in deep rolling $(\mathrm{mm})$

$d_{\mathrm{z}} \quad$ Thickness of heat-affected zone $(\mu \mathrm{m})$

$f \quad$ Helmholtz free energy $(\mathrm{J})$

$E \quad$ Electric field strength $\left(\mathrm{V} \mathrm{m}^{-1}\right)$

$e_{\mathrm{me}} \quad$ Stored mechanical energy $\left(\mathrm{J} \mathrm{mm}^{-3}\right)$

E. Brinksmeier

brinksmeier@iwt.uni-bremen.de

1 MAPEX Center for Materials and Processes, Leibniz Institute for Materials Engineering IWT, University of Bremen, Badgasteiner Str. 3, 28359 Bremen, Germany

2 Department of Civil Engineering, Institute of Applied Mechanics, RWTH Aachen University, Mies-van-der-RoheStr. 1, 52074 Aachen, Germany

3 Laboratory for Machine Tools and Production Engineering (WZL), RWTH Aachen University, Campus-Boulevard 30, 52074 Aachen, Germany

4 Institute of Aerodynamics, RWTH Aachen University, Wüllnerstr. 5a, 52062 Aachen, Germany $e_{\text {th }} \quad$ Experienced thermal energy $\left(\mathrm{J} \mathrm{mm}^{-3}\right)$

$h \quad$ Heat transfer coefficient $\left(\mathrm{W} \mathrm{m}^{-2} \mathrm{~K}^{-1}\right)$

$I_{\mathrm{e}} \quad$ Pulse current in EDM (A)

$k_{\mathrm{f}} \quad$ Flow stress (MPa)

$l_{\mathrm{g}} \quad$ Geometrical contact length $(\mathrm{mm})$

$t \quad$ Time (s)

$t_{\mathrm{c}} \quad$ Contact time (s)

$t_{\mathrm{e}} \quad$ Pulse duration in EDM $(\mu \mathrm{s})$

$v_{\mathrm{ft}} \quad$ Feed speed in grinding $\left(\mathrm{mm} \mathrm{min}^{-1}\right)$

$z \quad$ Depth below workpiece surface $(\mathrm{mm})$

$\varepsilon \quad$ Strain $(\mathrm{mm} / \mathrm{mm})$

$\dot{\varepsilon} \quad$ Strain rate $\left(\mathrm{s}^{-1}\right)$

$\varepsilon_{\mathrm{pl}, \mathrm{eq}} \quad$ Equivalent plastic strain $(\mathrm{mm} / \mathrm{mm})$

$\varepsilon_{\text {total,eq }}$ Total strain magnitude $(\mathrm{mm} / \mathrm{mm})$

$\vartheta \quad$ Temperature $\left({ }^{\circ} \mathrm{C}\right)$

$\sigma \quad$ Stress (MPa)

$\sigma_{\|} \quad$ Residual stress in feed direction (MPa)

$\sigma_{\mathrm{eq}} \quad$ von Mises equivalent stress (MPa)

$\sigma_{\mathrm{I}} \quad$ First-principal residual stress (MPa)

$\sigma_{\text {II }} \quad$ Second-principal residual stress (MPa)

$\varphi_{\text {ch }} \quad$ Chemical potential (J/mole)

$\varphi_{\mathrm{el}} \quad$ Electrode potential (V)

$\Delta x \quad$ Change of $x$ 


\section{Mathematical operator}

$\dot{x} \quad$ Time derivative of $\mathrm{x}$

$\nabla x \quad$ Spatial derivative of $x$

$\mathrm{d} x \quad$ Total derivative of $x$

$\max (x) \quad$ Maximum of $x$

mean $(x) \quad$ Arithmetic mean of $x$

\section{Abbreviations}

As Atomistic scale level

BVP Boundary value problems

CIRP International Academy for Production Engineering

ECM Electro-chemical machining

EDM Electric discharge machining

FEM Finite element method

FVM Finite volume method

FFT Fast Fourier transform

FP Ferritic/pearlitic

L Internal material load

LA Laser ablation

LCM Laser-chemical machining

M Material modification

MS Microstructural scale level

PC Polycrystalline scale level

PSC Process Signature Component

QT Quenched and tempered

RVE Representative volume element

\section{Introduction}

The prediction of subsurface properties after machining in terms of surface integrity has been an objective of researchers in the field of manufacturing technology for decades. However, even today desired material modifications, e.g., hardness, residual stress, microstructure and chemical composition, cannot be generated in prescribed tolerances with sufficient reliability. Especially for highly loaded components, the demand for an optimal state of material modifications and resulting optimal functional properties is a major challenge. In this paper, the underlying mechanisms leading to material modifications for specific manufacturing processes are investigated by means of analyzing the internal material loads. This mechanismbased view on surface integrity was introduced in the framework of "Process Signatures" in 2011 by Brinksmeier et al. [1]. Based on the description of the meaning and notation of a Process Signature, the papers' objective is to introduce individual Process Signature Components for processes with different predominant impacts. In particular, the major target of this work is to propose a mechanism-based approach to identify suitable descriptives of internal material loads for experimentally observed workpiece material modifications. Moreover, examples are given on how internal material loads can be determined by innovative in-process measurements and process simulations on the polycrystalline and microstructural scale level.

\section{Evolutionary View on Surface Integrity}

In the 1960s, researchers and practitioners in the field of manufacturing processes were mainly concerned about the geometrical properties of the finished component. The decision whether the component is capable of being used for the intended application or has to be sorted out or reprocessed only depended on the compliance with design tolerances. The cornerstones of manufacturing, published in 1956 by Kienzle [2], testify this geometry-driven view on the manufacturing processes. Modifications of the workpiece material due to the manufacturing process and their effect on the functional performance of the component instead, were not considered, neither by the manufacturer nor by the designer. In the following years, researchers like von Weingraber, Peters, Whitehouse and others identified the microgeometry, and with this the surface topography of the component, to be highly important for a variety of functional properties [3]. By paying more attention to the workpiece surface, more and more manufacturing-induced alterations in the surface area were considered by researchers. Finally in 1971, this development induced Field and Kahles [4] to release an extensive list of alterations and to define the term surface integrity for describing the condition of a surface after being generated. Since then, in addition to the geometrical properties, the state of surface integrity has been investigated by many researchers, concerned about the functional performance of manufactured components. In particular, changes in hardness, material structure and residual stress [5] were of major interest. In the 1980s, the importance of surface integrity was proven and the accumulated data showed that all manufacturing processes have certain effects on the workpiece material. With the increasing demands for highly stressed components, the request for a reliable prediction of the surface integrity emerged. A first approach toward a prediction of material modifications due to a process was presented by Malkin [6] in terms of grinding. His correlation of the specific energy with process parameters allowed the prediction of grinding burn. Brinksmeier [7] determined correlations of the changes of residual surface stress with the specific grinding power. By this, the prediction of residual surface stress, e.g., for different cutting speeds, could easily be calculated for the investigated grinding processes. Further investigations by Heinzel [8] considered the heat exposure time and led to a process window for grind hardening. Today, for selected processes, numerical [9-11] and analytical [12] models 
allow to choose suitable process parameters without iterative or experience-based knowledge. However, there is no commonly valid procedure to determine the required machining parameters for a given desired surface integrity in a knowledge-based way. This lack of knowledge was also confirmed within a collaborative work of CIRP from 2009 to 2011. Most of the participating research institutes were not able to generate a predefined surface residual stress of $-200 \mathrm{MPa}$ by a manufacturing process of their own choice [13]. The authors of this publication are convinced that this lack of knowledge results from the common process-oriented view in which the material modifications are directly correlated with the machining parameters. The internal material loads, e.g., stress, strain, temperature and temperature gradient, which actually lead to the observable modifications, are rarely or even not at all under consideration. As a consequence, the acquired correlations are mostly process-specific and cannot be transferred to modified or even other processes. Therefore, the objective of the 2011 coined term "Process Signature" for a new approach introduced in the following, and the 2014 established associated transregional Collaborative Research Center CRC/TRR 136 in Bremen, Aachen and Oklahoma is the systematic knowledge-based selection and configuration of manufacturing processes and their chains with regard to the desired surface integrity. Process Signatures correlate the material modifications with the internal material loads which lead to modifications by activating mechanisms such as yielding and phase transformations. It is expected that the utilization of Process Signatures should enable a comparability of apparently different machining processes, assuming that similar internal material loads will lead to similar material modifications. Consequently, Process Signatures are assumed to facilitate the solution of the so-called inverse problem in manufacturing [14].

\section{Process Signatures}

\subsection{Meaning and Notation}

In production engineering the need to generate predefined workpiece surface layer properties during machining is usually addressed by investigating the influence of the machining parameters on the resulting material modifications (Fig. 1, correlation A). Mostly, the determination of correlations of type A results in an extensive experimental study and the findings are only valid for the range of parameter values being investigated in that study. A more generalized description can be achieved by linking process quantities, e.g., process forces and machining power, to the resulting modifications (Fig. 1, correlation B). But still, the

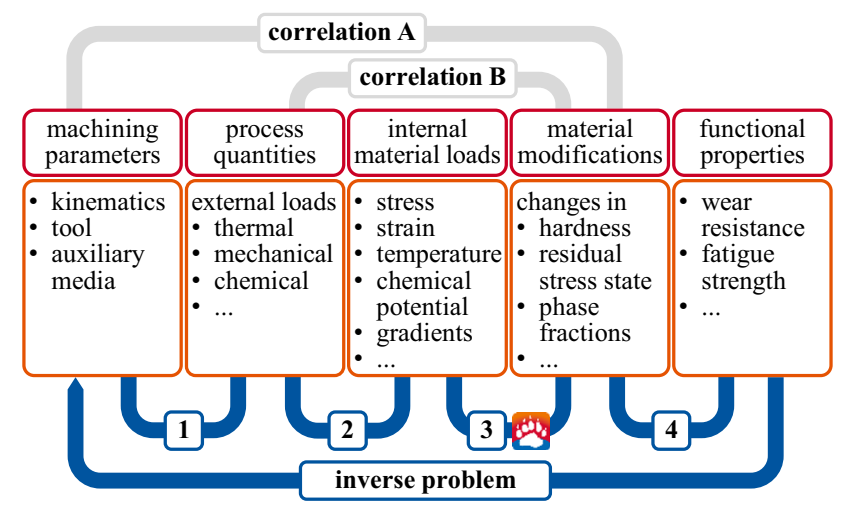

Fig. 1 Causal sequence of manufacturing processes [14]

underlying mechanisms of material modifications can only be described adequately when the internal material loads such as stress, strain, temperature and their respective gradients are taken into account. The correlation of these internal material loads with the resulting material modifications is proposed by the authors. In [1] the authors coined the term "Process Signature" for this correlation (Fig. 1, correlation 3).

Actually, Process Signatures need to be set up as a matrix, featuring single Process Signature Components (PSC) (Fig. 2). Each component contains a correlation between an internal material load $(L)$ and a material modification $(M)$ by means of the function $M=f(L)$. Thereby, the material modification has to be understood as the change of a workpiece surface layer property from an initial state to the resulting state after machining. Of particular interest are Process Signature Components (PSC) which have a relevance for the functional performance of the final part. Due to the objective to trace back the impact of the manufacturing process on the surface integrity to its causal mechanisms, Process Signatures have to be identified on the macro- and microstructural scale. Of course, these two scales are closely related to each other and the scaling within a Process Signature is an important objective for future work [14].

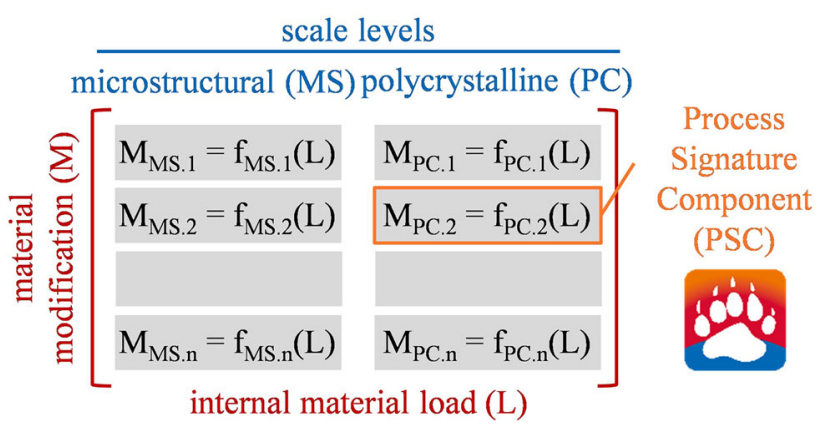

Fig. 2 Notation of a Process Signature with its single components [14] 


\subsection{Developing Process Signature Components}

During the development of a single Process Signature Component the most challenging task is to determine suitable descriptives of internal material loads of a manufacturing process. The question needs to be answered what are the causes of the material modification being observed in the experiment. By taking into account the underlying

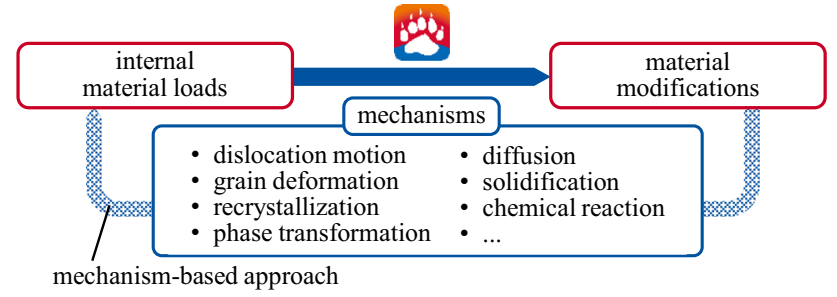

Fig. 3 Mechanism-based approach to determine relevant descriptives of internal material loads for process-specific material modifications

Table 1 Expected relevance of internal material loads on the polycrystalline scale for manufacturing processes according to their predominant impact on material modifications

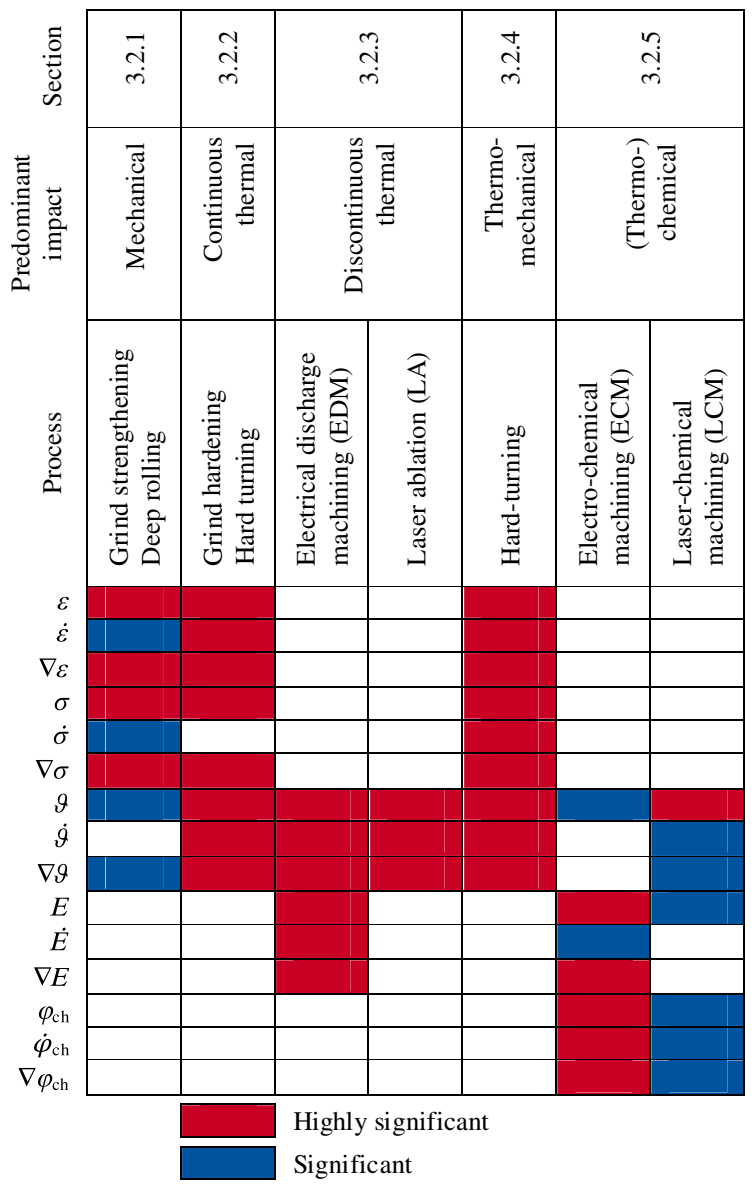

mechanisms in the material and, from there, discussing the reasons why these mechanisms are activated in the process, relevant internal material loads can be identified. In the following subsections, this mechanism-based approach, as shown in Fig. 3, is described for processes with different predominant impacts (Table 1) on workpieces made of the steel 42CrMo4 (AISI 4140) in two heat treatment conditions (FP and QT). Already established Process Signature Components are discussed from this perspective and novel descriptives of internal material loads and appropriate approaches for their determination are presented.

Besides the analysis of the main impacts (mechanical, thermal, chemical), novel findings regarding the combined impacts to the workpiece in manufacturing processes are also provided. As shown in Table 1, in this work processes with a predominantly thermo-mechanical impact and processes with a predominantly thermo-chemical impact, for instance, are investigated. Of particular interest in this regard is the grinding process due to its adjustability from a predominantly mechanical to a thermo-mechanical and up to a predominantly thermal impact by using appropriate machining parameters. To minimize the complex interactions of simultaneous thermal and mechanical effects, in this work, the grinding process purposely was designed in a way that led to a clearly predominantly thermal impact (grinding, grind hardening) or a predominantly mechanical impact (grind strengthening). Despite deviations from grinding processes established for industrial use, here the term grinding is used for grinding with a predominantly thermal impact without austenitization. In the future, the thermo-mechanical impact of grinding processes will also be investigated.

\subsubsection{Exemplary Process Signature Component for Processes with a Mechanical Main Impact}

When analyzing the internal material loads of processes with a mechanical main impact, the available Process Signature Components as presented by Meyer and Kämmler [15], Brinksmeier et al. [14] and Langenhorst et al. [16] focus on strains and strain fields in the subsurface layers. The investigations deal with deep rolling and grind strengthening (grinding with mechanical main impact). For both processes, the geometrical contact conditions and the acting contact forces exert a certain pressure on the workpiece surface. This causes specific stress and strain fields within the surface and subsurface layers which are hard to assess experimentally. Tausendfreund et al. [17] presented a speckle-based method to deduce stress and strain fields from local displacements of speckles during manufacturing processes. Furthermore, workpieces with integrated thin film sensors were developed. Meyer et al. [18] performed in situ X-ray diffraction experiments to 
analyze the stress fields in a deep rolling process. Promising results can be observed regarding these experimental approaches. However, analytically and numerically based assessments of the conditions during processes with a mechanical main impact also led to several Process Signature Components.

For processes with a mechanical main impact it can be assumed that the material modification is generated by plastic deformations due to yielding of the workpiece material. Yielding starts if an equivalent stress (e.g., von Mises stress) generated by the process reaches the yield strength. Hertz [19] derived equations to describe the mechanical loads in purely elastic bodies. With his equations combined with the maximum distortion energy theory by von Mises, equivalent stress distributions between interacting bodies can be predicted. This was done by Meyer and Kämmler to get a first impression whether equivalent stresses are suitable quantities to derive Process Signature Components for a deep rolling process. In [15], the authors correlated the analytically predicted maximum equivalent stresses $\max \left(\sigma_{\text {eq }}\right)$ with the maximum residual stresses measured after the process $\max \left(\sigma_{\|}\right)$. Despite some limitations (purely elastic assessment of the equivalent stresses in an elasto-plastic deep rolling process), they obtained a reasonable functional interrelation. This indicated that for processes with a mechanical main impact, strain and stress are, as expected, suitable quantities to represent the internal material loads. In particular, the results suggest that the complex tensorial stress field during the process can be characterized in a simplified way by a scalar value, namely the von Mises equivalent stress $\sigma_{\text {eq. }}$. Furthermore, residual stress seems to be sensitive and a significant material modification. The plastic deformations of the surface and subsurface layers inevitably cause distortions of the crystal lattice which manifest themselves in a change of the residual stress state. Thus, residual stress is ranked as an ideal material modification to focus on when it comes to correlating the internal material loads in processes with a mechanical main impact with changes of the surface and subsurface characteristics.

By utilizing Hertz' theory based on a purely elastic material behavior, the influence of work hardening on the yield strength cannot be taken into account. Moreover, regions of plastically deformed material affect the stress field generated by the tool-workpiece interaction. Therefore, the equivalent plastic strain $\varepsilon_{\mathrm{pl} \text {,eq }}$ was calculated in finite element simulations for different tool diameters $d_{\mathrm{b}}$ in a deep rolling process to characterize the internal material loads during the process (Fig. 4). This value includes the work hardening effect along the path of a volume element passing underneath the deep rolling tool. The correlations with the maximum change of residual stress (initial state

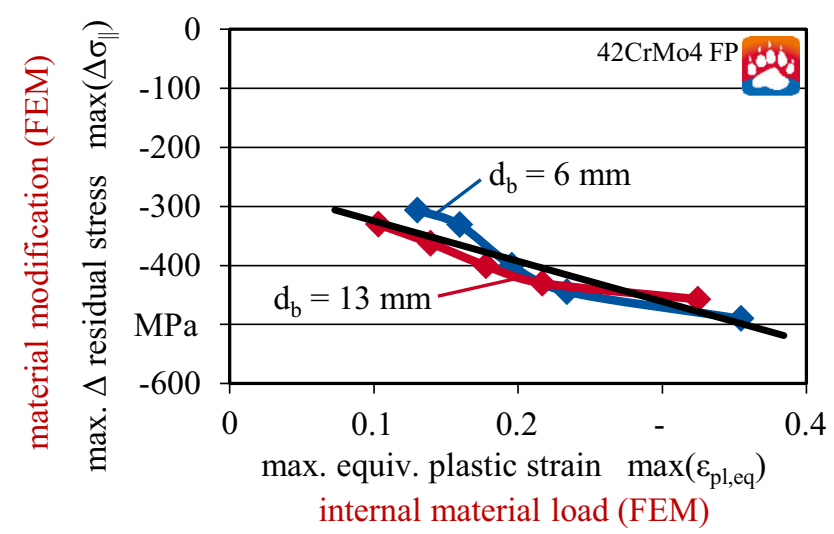

Fig. 4 Correlation of max. equivalent plastic strain and max. change of residual stress (PSC) for a deep rolling process [14]

assumed to be stress free) are very similar for both tool diameters and can be approximated by a linear function.

Langenhorst et al. [16] developed a new way to describe the mechanical loads caused by a single abrasive grain during grind strengthening by numerically modeling the contact with the workpiece as a moving normal and tangential pressure source. For the given contact conditions of a flattened single grain, the authors managed to reveal the interrelation between the maximum total strain magnitude $\max \left(\varepsilon_{\text {total,eq }}\right)$ (internal material load) and the maximum residual stress $\max \left(\sigma_{\|}\right)$(material modification). Also in this case the Process Signature Component for grinding observed by the authors is in good agreement with the ones presented in $[14,15]$ for deep rolling. This is another strong indication that Process Signatures have the potential to describe the effect of manufacturing processes in a mechanism-based and ideally in a process-independent way.

\subsubsection{Exemplary Process Signature Component for Processes with a Continuously Thermal Main Impact}

Since grinding is very adjustable, parameters can be found in such a way that the grinding process can be treated as a process with a mainly thermal impact. As a consequence the mechanical impact can be neglected, and it is acceptable to model the grinding process as a moving surface heat source. That was the assumption for the following analyses. Figure 5 shows two typical residual stress depth profiles depending on the maximum temperature $\max (\vartheta)$ during the process (initial state assumed to be stress free). If $\max (\vartheta)$ is smaller than the start temperature for austenite formation, residual tensile stress at the surface and two zero crossings below the surface can be observed. If $\max (\vartheta)$ is large enough to completely austenitize the near-surface volume and if additionally the cooling process is fast enough to create a martensitic layer at the surface, residual 


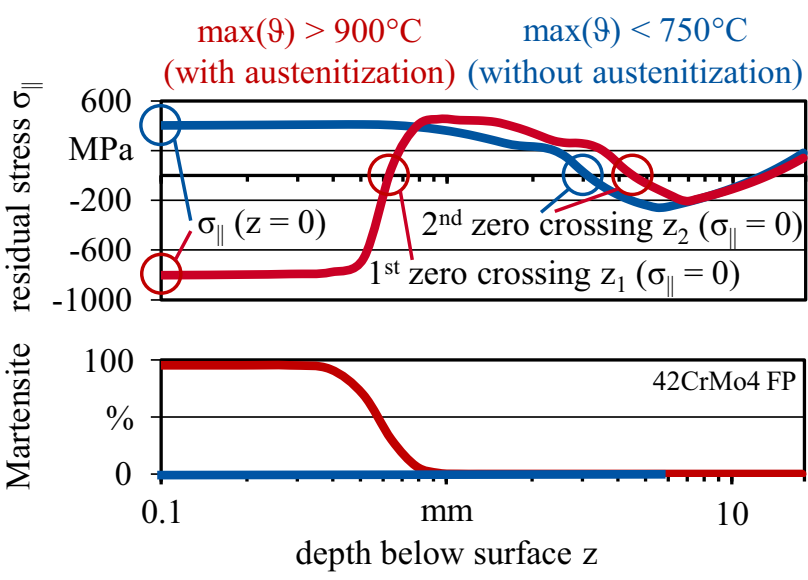

Fig. 5 Typical residual stress depth profiles and martensite distribution for grinding of bars with a rectangular cross section (based on FEM) acc. to [20]

compressive stress at the surface and an additional zero crossing result (Fig. 5). The depth of this zero crossing equals the depth of $50 \%$ martensite content [20]. As exemplary modification components of the corresponding Process Signature, the residual surface stress component parallel to the feed direction in grinding and the depth of zero crossings will be discussed in the following.

For the residual surface stress without martensitic transformation $\left(\max (\vartheta)<750{ }^{\circ} \mathrm{C}\right)$ it can be concluded from the analytical solution of the heat conduction equation that the maximum temperature and the maximum temperature gradient occur at the surface $(z=0)$ [21]:

$\max (\vartheta)=\vartheta(z=0)$

$\max (\nabla \vartheta)=\nabla \vartheta(z=0)$

This is true also for the maximum thermal strain and stress, since they are linked to the temperature and temperature gradients. If the temperature-dependent yield strength is not exceeded, no residual stress will be generated. For yielding a minimal (threshold) gradient is necessary. Above this critical gradient the residual stress at the surface increases with increasing values of $\max (\nabla \vartheta)$ (Figure 6, blue dots). Therefore, the maximum temperature gradient fulfills the above-mentioned condition for a relevant descriptive of the internal material load for grinding without phase transformation.

If a martensitic layer is formed, the resulting residual surface stress can be described by $\max (\nabla \vartheta)$, too (Fig. 6, red dots). But it has to be taken into account that for smaller values of this parameter the heat transfer coefficient $h$ and the maximum temperature have an influence. With increasing $\max (\nabla \vartheta)$ the resulting thickness of the martensitic layer decreases, because the minimal temperature for initiating the austenite formation moves closer to the surface. Therefore, the effects of martensite formation

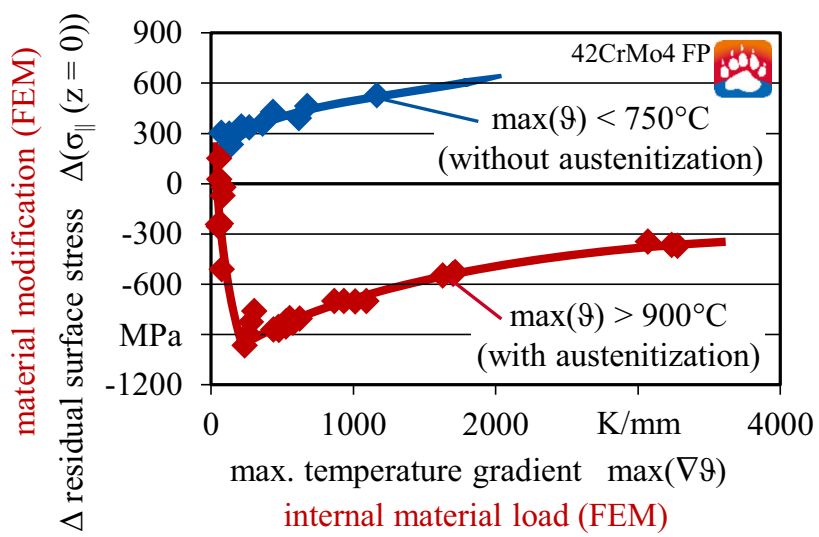

Fig. 6 Correlation of maximum temperature gradient and change of residual surface stress (PSC) for grinding acc. to [20]

decrease. At a maximum temperature gradient of $250 \mathrm{~K} / \mathrm{mm}$ a minimal residual surface stress is achieved. For higher values of the gradient the influence of this effect becomes smaller and smaller. Additionally, heat conduction from the surface to the bulk dominates more and more and subsequent self-quenching loses its influence.

Focusing now on the depth of zero crossings, the analytical solution of the heat conduction equation gives again hints for the choice of suitable descriptives of internal material loads. Having in mind that the depth of the first zero crossing is equal to the depth of $50 \%$ martensite content, it seems reasonable to ask in which way the depth of the transformation start temperature $A_{\mathrm{c} 1}$ depends on the thermal loads at the surface. For a constant value of a maximum temperature increase the depth of transformation start is proportional to the square root of the contact time $t_{\mathrm{c}}$ :

$\Delta \vartheta=$ const. $\rightarrow z\left(A_{\mathrm{c} 1}\right) \sim \sqrt{t_{\mathrm{c}}}, \quad t_{c}=l_{\mathrm{g}} / v_{\mathrm{ft}}$

For a constant contact time this depth depends linearly on the maximum temperature difference:

$t_{\mathrm{c}}=$ const. $\rightarrow z\left(A_{\mathrm{c} 1}\right) \approx b_{1} \cdot \max (\Delta \vartheta)+b_{2}$

Hence, as the simplest approach for a descriptive of the internal material load the product $\max (\Delta \vartheta) \cdot \sqrt{t_{\mathrm{c}}}$ was utilized. This term is proportional to the total energy input by the grinding process [21]. Figure 7 shows the correlations for the first two zero crossings of the residual stress depth profiles. All values of the second zero crossing (tensile to compressive) can be approximately described by one curve independent of the maximum temperature reached during the process. The only precondition is that $\max (\nabla \vartheta)$ must be larger than $100 \mathrm{~K} / \mathrm{mm}$. This means that for this component of the Process Signature $\max (\Delta \vartheta) \cdot \sqrt{t_{\mathrm{c}}}$ is a good choice for characterizing the internal material load.

The values of the first zero crossing (compressive to tensile) shows in principle a linear trend but with a superimposed variation. Therefore, this approach for a 


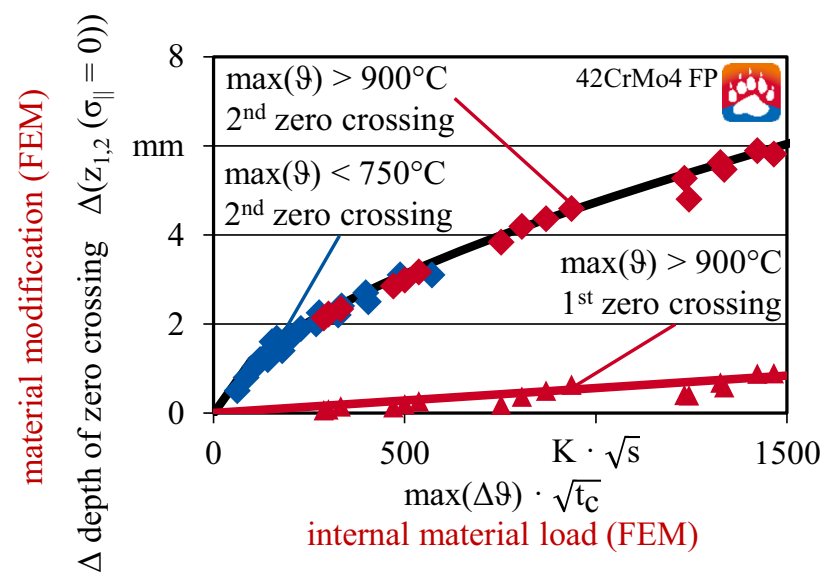

Fig. 7 Correlation of $\max (\Delta \vartheta) \cdot \sqrt{t_{c}}$ at different maximum temperatures and change of depth of zero crossing of the residual stress depth profile (PSC, valid if $\max (\nabla \vartheta)>100 \mathrm{~K} / \mathrm{mm}$ ) for grinding acc. to [20]

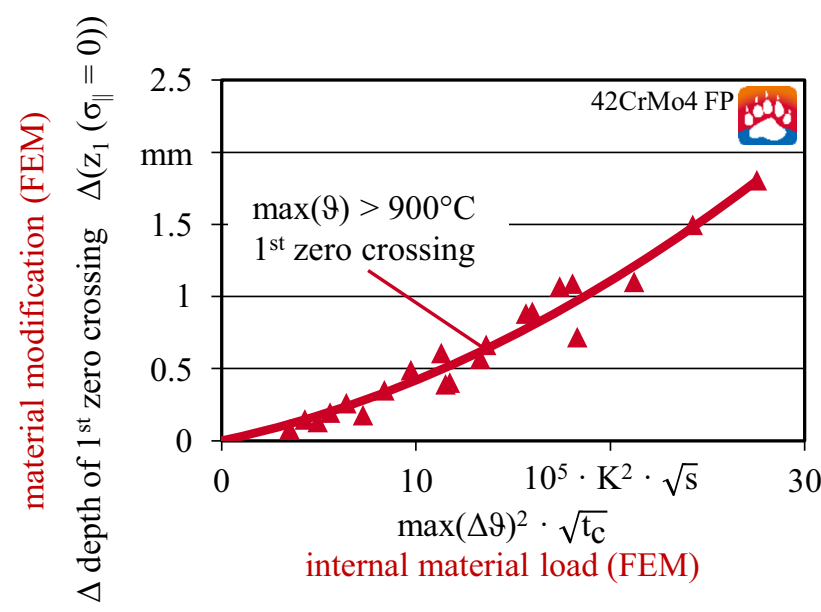

Fig. 8 Correlation of $\max (\Delta \vartheta)^{2} \cdot \sqrt{\mathrm{t}_{\mathrm{c}}}$ and change of depth of the first zero crossing of the residual stress depth profile (PSC, valid if austenitization occurs) for grinding acc. to [20]

descriptive of the internal material load is acceptable but not optimal. The reason is that the analytical solution of the heat conduction equation does not include phase transformations. But as mentioned before the depth of this zero crossing equals the depth of $50 \%$ martensite content. For the formation of martensite at least an austenitization is necessary. This means that an increasing depth of a $50 \%$ martensite content requires an increased depth of austenite formation. This can be fulfilled by an increased maximum temperature. Therefore, an additional term $\max (\Delta \vartheta)$ should represent this missing effect in a more suitable descriptive of the internal material load. Figure 8 shows the depth of the first zero crossing as a function of the descriptive $\max (\Delta \vartheta)^{2} \cdot \sqrt{t_{c}}$ of the internal material load. This component of the Process Signature for a process with a continuously thermal main impact such as grind hardening can be described by a parabolic function. The quality of this fit is better than the first approach if the different scales of Figs. 7 and 8 will be taken into account.

\subsubsection{Exemplary Process Signature Component for Processes with a Discontinuously Thermal Main Impact}

The Process Signature Components of two machining processes with a discontinuously thermal main impact are considered in this section, i.e., electrical discharge machining (EDM) and laser ablation (LA). With both processes material is removed from the workpiece surface due to a local temperature reaching values above the melting or even the evaporation point of $42 \mathrm{CrMo} 4$ (AISI 4140). Single sparks (EDM) or laser pulses (LA), with a repetition rate in the order of $100 \mathrm{kHz}$, are used to remove the workpiece material. In the EDM process usually a dielectric liquid fluid such as oil or deionized water is used, in which the spark propagates from the electrode to the workpiece. The short impact of the high electric current or the energetic laser pulse strongly heats up the surface such that it melts and possibly evaporates.

The determination of a Process Signature requires a suitable descriptive of the internal material load for a specific material modification. The chemical load in form of the dielectric fluid can be responsible for carbon diffusion into the material such that a slightly higher residual austenite concentration can be observed in the workpiece. In both machining processes, however, the thermal load can be considered as the main impact, which has a strong influence on the residual stress, the hardness and ultimately on the fatigue limits. The local high power of the pulses leaves a volume of liquid material, which partly is removed and partly resolidifies. Beneath the resolidified layer a heataffected zone, in which phase transformation occurs, is generated. Since the temperature distribution varies strongly in space and time, a suitable descriptive of the internal material load has to be determined. Direct temperature measurements are typically not feasible due to the remarkable tiny spatial $(\mu \mathrm{m})$ and temporal $(\mu \mathrm{s})$ scales. Therefore, the temperature field in the workpiece must be determined by a simulation approach, which needs to assume an energy input of the laser pulse in LA or the spark in EDM. This energy input, however, is not exactly known. Therefore, an approximate energy amount, absorbed by the workpiece, has to be assumed. For the EDM process, investigations have shown that an energy fraction of about $50 \%$ going into the workpiece seems to be a good assumption [22].

In Fig. 9 the residual surface stress is plotted as a function of the average pulse temperature at the workpiece 


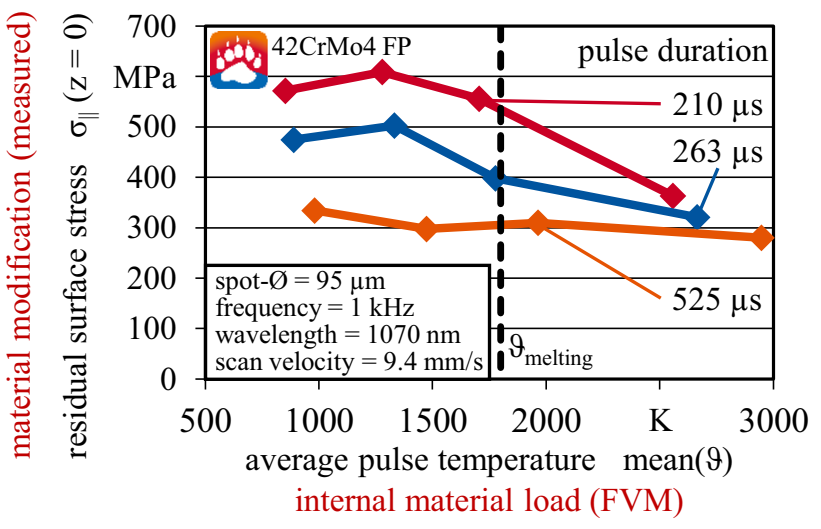

Fig. 9 Correlation of average pulse temperature at the surface and residual surface stress for laser ablation with various pulse durations

surface for various pulse durations in a laser ablation process. The different curves clearly indicate that the residual surface stress is not sufficiently described by the average temperature. From that it can be deduced that a more suitable descriptive of the internal material load is needed. In order to provide the same pulse energies in the experiments higher pulse powers were utilized for shorter pulse durations. In micrographs of the workpiece material it was observed that the shape of the molten and resolidified material volume depends on the pulse power: for small powers, the resolidified workpiece material has a semispherical shape, which turns into a cone-like shape with higher pulse power. It is assumed that this affects the maximum temperature at the surface as well as the phase transformation mechanisms and consequently the generation of residual stress. Future work will take this mechanism into account to further develop the Process Signature for laser ablation.

First results of a Process Signature Component were obtained with the mean maximum temperature gradient in $z$-direction mean $(\max (\nabla \vartheta))$ for EDM as a suitable descriptive of the internal material load (Fig. 10). The temperature in the EDM process has been determined with simulations using the FEM model described by Klocke et al. [23]. A clear correlation between the thickness of the heat-affected zone and the averaged maximum temperature gradient can be observed in Fig. 10. The mean maximum temperature gradient is obtained by averaging the maximum temperature gradient in $z$-direction occurring during the pulse over the first micrometer below the melting pool boundary. The results for the simulated heat-affected zone are in good agreement with the experimental data obtained by Klocke et al. [24]. This correlation confirms the applicability of the Process Signature concept to processes with discontinuously thermal main impact and confirms that it is possible to predict the material modification from the simulated internal material load.

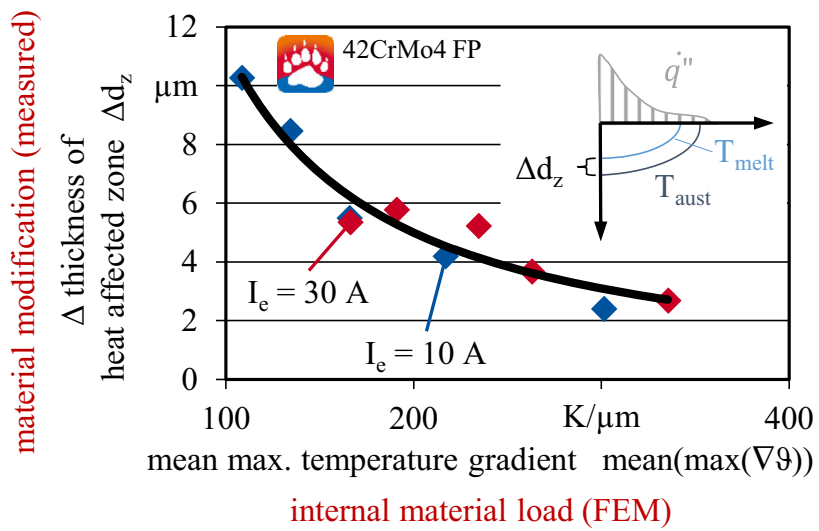

Fig. 10 Thickness of the heat-affected zone as a function of mean maximum temperature gradient on the first micrometer beneath the melting boundary for varying discharge currents

The present investigations only consider single-pulse effects. Future work will concentrate on the analysis of the cumulative effect of multiple pulses. Additionally, a refined model for the material modification will be developed with the aim to identify the most suitable descriptives of the internal material load for Process Signatures. First approaches were already obtained and published by Klocke et al. [25].

\subsubsection{Exemplary Process Signature Component for Processes with a Thermo-Mechanical Impact}

Most of the manufacturing processes involving primarily mechanical impacts can in fact not be addressed as pure mechanical processes only. Looking on structural or even atomistic level of matter, internal mechanical loads will also involve and evoke a thermal response of the material. Under certain conditions the thermal impact may be neglected and processes be treated like ones with a mechanical main impact, but in principle the thermal impact has to be accounted for, and depending on the nature of the process itself, processes with mechanical impact may even be governed by thermal effects. With respect to modeling of Process Signature Components both impacts mechanical and thermal may be treated separately first and then combined by superposition. But moreover both impacts are influencing each other: for instance internal thermal loads can be generated through mechanical impacts and, on the other hand, thermal loads are altering the mechanical properties and consequently the elastoplastic material behavior. Some material modification mechanisms only occur if both mechanical and thermal impacts occur simultaneously, e.g., dynamic recrystallization. Thus, a far more sophisticated approach is needed to identify the relevant descriptives of the internal material loads. 
So far for this kind of processes a number of different experimental and modeling efforts have been undertaken to analyze the thermo-mechanical behavior of steel in cutting processes. Common experimental methods have been applied to measure external material loads in-process [26], and new techniques have been developed to measure simultaneously the internally acting mechanical and thermal loads as well [27]. These in-process measurements together with intense post-process material characterization of microstructure, hardness and residual stress are applied to analytical and numerical process models for further analysis and comprehension of the underlying mechanisms of the material modification.

The analysis of microstructural modifications in metal cutting of steel, i.e., the formation of a white layer, leads to a first Process Signature Component based on process simulations [28]. For this purpose a new thermodynamic model was developed by Buchkremer and Klocke. The model describes the grain size as a function of the descriptives of the internal material loads temperature $\vartheta$, specific mechanical energy $e_{\text {me }}$ (plastic work) and the total derivative of the Helmholtz free energy $d f$. According to this model dynamic recrystallization takes place if the temperature and the mechanical energy reach a critical value and if simultaneously the total derivative of the Helmholtz free energy is negative. The predicted grain size distribution and the thickness of the affected boundary layer agree well with measurements using transmission electron microscopy. The model was further on used to correlate the internal material loads in hard turning with the generated (maximal principal) residual stress in the machined surface layer measured by $\mathrm{X}$-ray diffraction (Fig. 11).

Within well-defined limits of the stored mechanical energy $e_{\mathrm{me}}$ and the experienced maximum thermal energy $\max \left(e_{\mathrm{th}}\right)$ specific areas for residual stress can be obtained. The mechanical energy $e_{\mathrm{me}}$ is the plastic work done on a

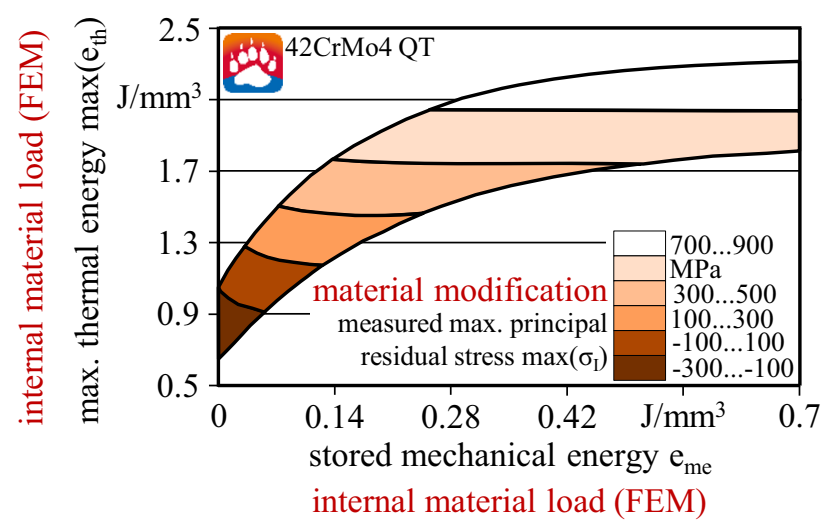

Fig. 11 Process Signature Component (PSC) for hard turning (orthogonal cutting) acc. to [30] volume element of the workpiece material and is a function of the strain $\varepsilon$ and the flow stress $k_{\mathrm{f}}$. The flow stress $k_{\mathrm{f}}$ itself is a function of the temperature $\vartheta$, the strain $\varepsilon$ and the strain rate $\dot{\varepsilon}$. Therefore, the mechanical energy is a highly aggregated quantity to describe the mechanical impact of the internal material load taking into account the influence of strain hardening during the process. The results indicate its usefulness in describing the mechanical impact of a thermo-mechanical process. The maximum thermal energy $\max \left(e_{\mathrm{th}}\right)$ is proportional to the maximum temperature $\vartheta$ of the volume element and takes into account the temperaturedependent thermophysical material properties. The Process Signature Component shows that when the thermal energy falls below a certain limit, the mechanical impact starts dominating the resulting material modifications leading to residual compressive stress. With increasing mechanical energy also more heat is generated during the process, ultimately leading to residual tensile stress in the workpiece surface layer [28, 29].

As outlined above the mechanism of dynamic recrystallization can lead to white layers when machining steel. Besides the governing temperature also strain and strain rate are the decisive parameters of this mechanism. But also other mechanisms can lead to a reduction of grain size, e.g., phase transformation; here temperature is crucial as well, but additionally the duration of the temperature impact is determining the resulting material modification. To distinguish these modifications and their underlying mechanisms further investigations, characterization and modeling are necessary to fully understand the influence of thermo-mechanical impacts of machining processes on the elasto-plastic material behavior and the resulting material modification. In addition the analyses presented here addressed primarily the polycrystalline level. In order to fully understand and adequately describe the mechanisms leading to material modifications within the framework of Process Signatures it is necessary to extend the modeling and simulation approaches to the microstructural level as presented in Sect. 4.

\subsubsection{Exemplary Process Signature Component for Processes with a Chemical and Thermo-Chemical Impact}

Processes with a predominantly chemical and thermochemical impact-like electrochemical machining (ECM) and laser-chemical machining (LCM) - include the internal material loads of local chemical potential $\varphi_{\mathrm{ch}}$, electric field strength $E$ and temperature $\vartheta$. Besides that, also the local mechanical surface tensions resulting from fluid-structure interactions have to be considered as an important loading for high fluid flow rates. By the main underlying mechanism of local chemical reaction in terms of anodic metal 
dissolution on the atomistic scale different resulting workpiece surface layer modifications can therefore be identified especially on the microstructural scale [31]. For both above-mentioned processes these modifications include changes of the chemical composition and of complete phase fractions (e.g., oxide and passivation layer formation) at the surface as well as the formation of surface topography and also porosity due to pitting corrosion and selective dissolution [32].

The physical causes of the internal material loads differ considerably for LCM and ECM. During the first process, the material is heated locally and chemical reactions are therefore thermally activated. In ECM the material is dissolved by an electric field support [33]. From a superordinate point of view the necessary energy for initiating chemical reactions can be provided in both ways thermally or electrically or even by a combination of them [33]. For both processes the formation of an initial passivating layer is very important to avoid excessive and uncontrolled general dissolution and allowing the distinct transpassive material removal when locally increasing the according material loadings. Therefore, in Fig. 12 the generation of a passive layer-as a process inherent mechanism-is shown for both processes as a function of both internal material loads, electric field strength $E$ and temperature $\vartheta$. The electrode potential $\varphi_{\mathrm{el}}$ as an aggregated parameter (y-axis) represents the line integral of the local electric field strength over the electrolytic double layer on the anodic workpiece surface along the electric field lines.

The graph represents a first Process Signature Component for processes with chemical and thermo-chemical impact in a general form and especially independently of the individual process technologies and machine tool settings. It distinguishes between areas with stable and unstable passive layer formation for local loadings of temperature and electrode potential for a given workpiece

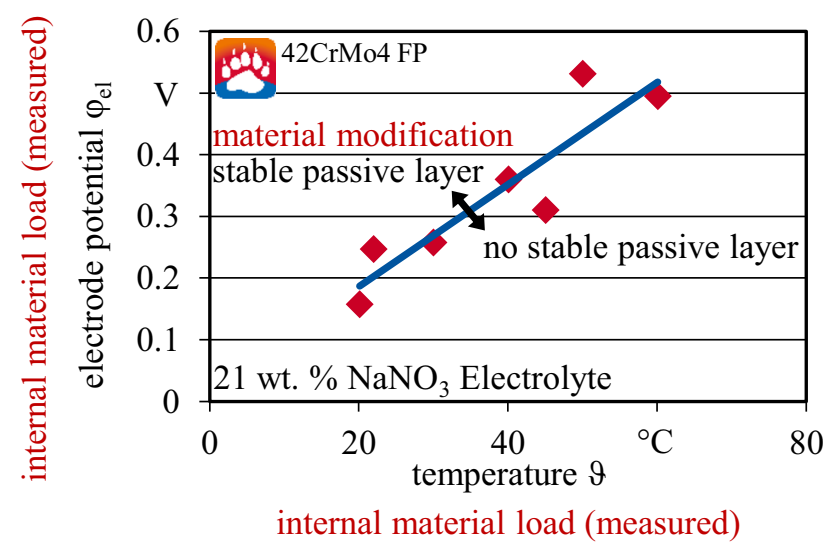

Fig. 12 Passive layer formation as a function of temperature and electrode potential (PSC) for a given chemical potential during LCM and ECM material and chemical potential $\varphi_{\mathrm{ch}}$ defined by the used electrolyte. In this specific case, it can clearly be seen that for LCM (generally applied at room temperature) an additional electrode potential is necessary to achieve a sufficient surface passivation. For locally increased temperatures caused by the laser beam machining can be performed and controlled keeping the electrode potential constant. For ECM at elevated temperatures also the required minimum electrode potential can be identified.

A second example for a Process Signature Component deduction for electrochemical processes is shown in Fig. 13. Based on specific material removal rates of the material phases involved and temporarily and spatially evolving local electric field strength during the process, a comprehensive modeling on the microstructural scale was carried out $[34,35]$. As a result, the change in the phase fraction of cementite and ferrite can be seen as a function of the maximum electric field strength as the relevant descriptive of the internal material load. The cause for these changes is given by the different selective dissolution behaviors of the metallic phases. For comparatively low field strengths $E$ - typically in ECM side gaps-a faster dissolution of ferrite results in a negative change of this phase at the surface due to the formation of surface ditches and valleys. Therefore, the fraction of cementite increases resulting in a rough surface roughness and likely a changed local hardness distribution (still to be analyzed). For high electric field strengths $E$ - typically in the frontal machining gap —no significant changes occur. The results agree well with experimental results, therefore validating the given modeling approach [35].

Future modeling approaches for Process Signature Components of processes with chemical and thermochemical impact will focus on a more comprehensive energy-based description based on the Gibbs free energy, cf. [33]. It is assumed that this will allow a persistent modeling approach also enabling inverse approaches to deduce appropriate process parameter settings to achieve

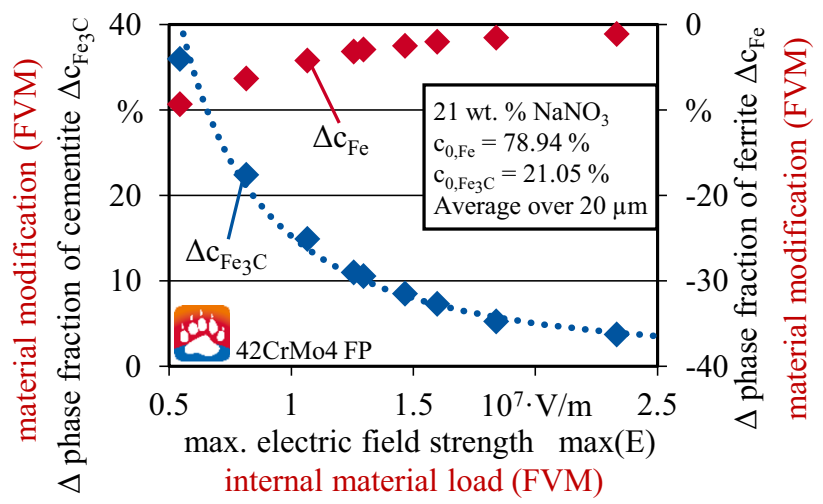

Fig. 13 Correlation of max. electric field strength and change of phase fractions (PSC) for ECM acc. to [35] 
the desired surface integrity of workpieces. This will finally allow to reach the targeted part functionalities [36] of chemically and thermo-chemically treated parts in a defined way.

\subsubsection{Interim Conclusion}

The exemplary discussions for different classes of manufacturing processes show that the approach of building a logical chain from the observed material modification via the underlying mechanisms to the internal material load is very promising to systematically develop Process Signature Components.

In particular this mechanism-based approach facilitates the identification of suitable descriptives for the processspecific internal material loads as shown in Table 2. The following conclusions can be drawn from the presented analyses:

- A simplified quantification of the complex timedependent and spatially varying fields of the stress and strain tensor seems to be feasible. For processes with a mechanical main impact equivalent stresses and/ or strains as descriptives for the internal material loads result in plausible Process Signature Components. This observation is highly important since a reduction of complexity is essential to utilize Process Signatures in practical applications which is the ultimate goal of the presented research.
- For processes with a thermal main impact the heat exposure time needs to be taken into account. For example the thickness of the heat-affected zone depends on the temperature increase and on the duration of the temperature increase. Due to the diffusive nature of heat conduction in solids the square root of the time is the decisive quantity that also should be taken into account (c.f. Figure 7).

- Energy-based quantities should be taken into account if they are required for an adequate description of the material modification mechanism. For example, in hard turning the onset of dynamic recrystallization, which is in this case the mechanism for the formation of a white layer, depends on three descriptives: simultaneously the mechanical and thermal energy must surpass a critical value and the total derivative of the Helmholtz free energy must be smaller than zero. This example also implies that a comparatively simple graphical representation of Process Signature Components is not always possible.

- In some cases the material modification depends on the interaction of internal material loads. For example for the formation of a passivation layer in ECM the minimum required electric field strength depends on the temperature. This dependence should be reflected by the notation of the relevant descriptives, e.g., for the above-mentioned case: $\vartheta, E(\vartheta)$.

Table 2 Summary of discussed descriptives of internal material loads as a result of the mechanism-based approach

\begin{tabular}{|c|c|c|c|}
\hline Impact & Material modification & Mechanism & $\begin{array}{l}\text { Descriptive of the internal } \\
\text { material load }\end{array}$ \\
\hline Mechanical & - $\max \left(\Delta \sigma_{\|}\right)$ & $\begin{array}{l}\text { - Yielding } \\
\text { - Strain hardening } \\
\text { - Strain rate hardening }\end{array}$ & $\begin{array}{l}\text { - } \max \left(\sigma_{\mathrm{eq}}\right) \\
\text { - } \max \left(\varepsilon_{\mathrm{pl}, \mathrm{eq}}\right) \\
\text { - } \max \left(\varepsilon_{\mathrm{total}, \mathrm{eq}}\right)\end{array}$ \\
\hline Continuously thermal & $\begin{array}{l}\text { - } \Delta\left(\sigma_{\|}(z=0)\right) \\
\text { - } \Delta\left(z\left(\sigma_{\|}=0\right)\right)\end{array}$ & $\begin{array}{l}\text { - Yielding } \\
\text { - Strain hardening } \\
\text { - Phase transformation }\end{array}$ & $\begin{array}{l}\text { - } \max (\vartheta) \\
\text { - } \max (\nabla \vartheta) \\
\text { - } \max (\Delta \vartheta) \cdot \sqrt{\mathrm{t}_{\mathrm{c}}} \\
\text { - } \max (\Delta \vartheta)^{2} \cdot \sqrt{\mathrm{t}_{\mathrm{c}}}\end{array}$ \\
\hline Discontinuously thermal & $\begin{array}{l}\text { - } \Delta \mathrm{d}_{\mathrm{z}} \\
\text { - } \sigma_{\|}(z=0)\end{array}$ & $\begin{array}{l}\text { - Solidification } \\
\text { - Phase transformation }\end{array}$ & $\begin{array}{l}\text { - } \max (\nabla \vartheta) \\
\text { - } \operatorname{mean}(\vartheta)\end{array}$ \\
\hline Thermo-mechanical & $\begin{array}{l}\text { - } \max \left(\sigma_{\mathrm{I}}\right) \\
\text { - } \max \left(\sigma_{\mathrm{II}}\right) \\
\text { - Average grain size }\end{array}$ & $\begin{array}{l}\text { - Yielding } \\
\text { - Strain hardening } \\
\text { - Strain rate hardening } \\
\text { - Thermal softening } \\
\text { - Dynamic recrystallization }\end{array}$ & $\begin{array}{l}\text { - } e_{\mathrm{me}} \\
\text { - } e_{\mathrm{th}} \\
\text { - } \max \left(e_{\mathrm{th}}\right) \\
\text { - } d f \\
\text { - } \vartheta\end{array}$ \\
\hline (Thermo-) chemical & $\begin{array}{l}\text { - Passivation layer formation } \\
\text { - } \Delta \text { phase fractions }\end{array}$ & - Chemical reaction & $\begin{array}{l}\text { - } \varphi_{\mathrm{el}} \\
\text { - } \varphi_{\mathrm{ch}} \\
\text { - } \vartheta \\
\text { - } \max (E)\end{array}$ \\
\hline
\end{tabular}


- With the exception of ECM mechanisms were solely analyzed on the polycrystalline scale for which mostly phenomenological models were utilized. Simulations on the microstructural scale would allow a more realistic description of the mechanisms involved and may therefore lead to more suitable descriptives of the internal material loads (c.f. Sect. 4).

- The Process Signature Components presented in this paper are based on a specific workpiece material (42CrMo4) heat-treated in two different ways: tempered to a ferritic/pearlitic microstructure and quenched and tempered. If other initial microstructures or other steel grades will be used, it cannot be assumed that the correlations found between internal material loads and modifications will remain unchanged. In this case the relevant material properties have to be integrated into the descriptives of the internal material loads.

In order to develop Process Signatures a detailed knowledge about the internal material loads is required. Due to technological limitations they are not accessible by the available techniques in a broad range of process conditions. Consequently, the internal material loads and their suitable descriptives presented here were mostly determined in numerical simulations. Nevertheless, new measurement techniques for quantifying internal material loads during the manufacturing process are developed aiming at a validation of the simulation results and the underlying models for specific process conditions.

\section{Finding Relevant Internal Material Loads by Modeling on Different Scales}

The microstructure of most materials of technological importance is characterized by a complex distribution of individual grains and phase constituents which might vary in their size, morphology and orientation. Depending on the process-induced material load, this microstructure evolves (e.g., by martensitic phase transformations [37] or dynamic recrystallization [38]) which influences not only the physical, topological and statistical characteristics of the microstructure but also the workpiece material behavior during the process. Since such process-microstructureproperty relations and the resulting Process Signature Components [30, 39] cannot be established based on experimental data only, numerical methods are often employed.

In order to capture microstructural features as well as the process modeling, scale separation might be assumed and two-scale models be employed. Scale separation means that the two considered scales-here the microstructural and the polycrystalline levels-are sufficiently different

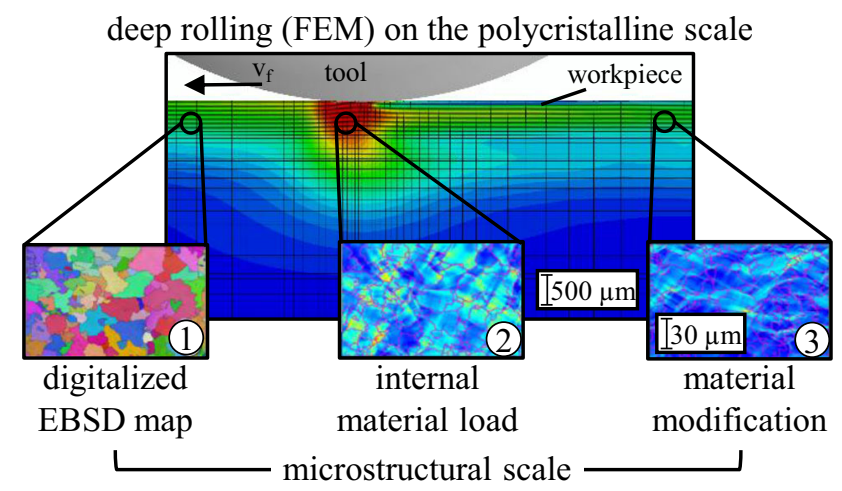

Fig. 14 Schematic of a two-scale computational process modeling of polycrystalline materials

from each other. Most common in the context of computational homogenization methods [40] is the multilevel finite element (FE) or $\mathrm{FE}^{2}$ method [41]. In this case the microstructure is represented by a representative volume element (RVE) [42] and attached to each integration point of the discretized workpiece material (Fig. 14). At both scales, the microstructural and polycrystalline scale, the FE method is exploited to solve the resulting two locally coupled boundary value problems (BVP). The thermomechanically coupled BVPs consist of the balance of linear momentum and energy balance which have to be solved in an iterative fashion. The handshake between both scales is based on appropriate volume-averaging concepts [43].

Although the $\mathrm{FE}^{2}$ method represents a flexible and wellestablished numerical tool, high-fidelity two-scale simulations of complex processes with heterogeneous microstructures are correlated with an excessive computational effort. Therefore, recently, FE-FFT-based methods [44-46] have been developed which represent a powerful and an efficient alternative to the classical $\mathrm{FE}^{2}$ method. In this case the BVP at the microstructural scale is solved using fast Fourier transforms (FFT) and fixed-point methods [47]. Different authors [48] have shown that such FFT-based numerical schemes are computationally more efficient than FE-based approaches. Focusing on polycrystalline materials with elasto-viscoplastic constitutive behavior, small [46] and finite strain [49] two-scale models have been developed as well as phase-field modeling of martensitic phase transformations [45]. For the elasto-viscoplastic constitutive model, the two-scale FE-FFT-based approach has qualitatively been validated based on experimental three-point bending tests [46].

\subsection{Modeling on the Microstructural Scale Level}

As alluded above, the balances of linear momentum and energy are solved in an iterative fashion at the microstructural scale yielding the local displacement field 

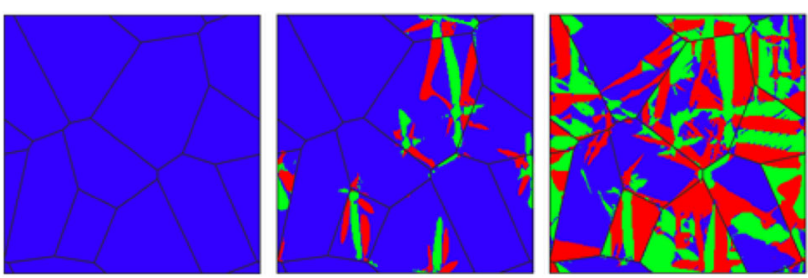

Fig. 15 Example of phase-field modeling of martensitic phase transformations in metals [45]

and temperature distribution. Based on the kinematic and constitutive assumptions, the strain and stress fields as well as the heat flux are computed. In order to account for microstructural evolution, additional field variables are introduced. These are the so-called phase fields or nonconserved order parameters. The latter describe the spatial distribution and volume fraction of the different phase constituents. The evolution of the phase fields is governed by the Allen-Cahn equation which minimizes the total free energy of the system. Note that the special form of the free energy density defines the phenomenon to be modeled and represents the coupling between the thermo-mechanical problem and the evolution of the microstructure.

An example for phase-field modeling of martensitic phase transformations is shown in Fig. 15, where the parent austenite phase is characterized by blue and two martensite variants in green and red, respectively. An overview about various types of phase-field models and recent advantages in associated numerical methods can be found in [50].

\subsection{Transfer between Microstructural and Polycrystalline Scale Level}

At the polycrystalline scale the FE method is employed to determine the nodal displacements and temperature. In each integration point the displacement gradient and heat flux are computed and imposed to the RVE. The FFT-based solution of the BVP at the microstructural scale leads to the heterogeneous displacement and temperature field distribution. Homogenization of the latter quantities leads to the effective thermo-mechanical constitutive response of the microstructure. For the mechanical part, the homogenization procedure is simply given through the volume average of the microstructural stress distribution [49]. Appropriate volume-averaging concepts for the thermal part have been proposed by [51]. This means that the transfer between both scales only requires the transfer of the displacement gradient and temperature and the computation of the homogenized constitutive RVE response. Internal variables associated with dissipative processes (e.g., plasticity, damage) as well as the order parameters are solely defined at the microstructural scale and have to be stored at each integration point at the polycrystalline scale for each loading step. Thus, two-scale simulations of technologically relevant processes (e.g., deep rolling, metal cutting) represent still a tremendous challenge. The improvement of the micromechanical Fourier spectral solver and development of model order reduction techniques for FFT methods are subject of current research and might increase the efficiency of two-scale full-field simulations tremendously. Then, numerical and experimental results of complex processes can be compared not only qualitatively but also quantitatively. On this basis, internal material loads and process-induced modifications are accessible on both scales and can be used for a detailed analysis and the establishment of Process Signature Components.

\section{Summary and Outlook}

This paper summarizes the current state of the art in developing and determining Process Signatures in a mechanismbased way and gives examples of Process Signature Components for different processes. The concept was proven for the investigated cases, varying in the mechanical, thermal and chemical impact on the workpiece material. The major challenge of determining the significant descriptives of the internal material loads for a specific material modification is presented for all cases. Within this approach a deep understanding of the underlying mechanisms can be established. For this objective, the paper shows how modeling on different scales can make substantial contributions. In future work, these approaches will help to consider the impact of the initial material state of the workpiece material and the impact of multistage processing and process chains in the framework of Process Signatures.

Acknowledgements The authors wish to thank the German Research Foundation (DFG) for funding this work within the transregional Collaborative Research Center SFB/TRR 136 "Process Signatures", subprojects M01, M03, M04, M05, F01, F02, F03, F05, F06. Also, the valuable contributions of F. Frerichs (Leibniz Institute for Materials Engineering IWT), S. Eckert (BIAS-Bremer Institut für angewandte Strahltechnik) and S. Harst (Laboratory for Machine Tools and Production Engineering WZL) are gratefully acknowledged.

Open Access This article is distributed under the terms of the Creative Commons Attribution 4.0 International License (http://creative commons.org/licenses/by/4.0/), which permits unrestricted use, distribution, and reproduction in any medium, provided you give appropriate credit to the original author(s) and the source, provide a link to the Creative Commons license, and indicate if changes were made.

\section{References}

1. Brinksmeier E, Gläbe R, Klocke F, Lucca DA (2011) Process signatures - an alternative approach to predicting functional workpiece properties. Proced Eng 19:44-52 
2. Kienzle O (1956) Die Grundpfeiler der Fertigungstechnik. VDIZeitschrift 98(23):1389-1395

3. Whitehouse DJ, Vanherck P, de Bruin W, van Luttervelt CA (1974) Assessment of surface topology analysis technique in turning. Ann CIRP 23(2):265-282

4. Field M, Kahles JF (1971) Review of surface integrity of machined components. CIRP Ann Manuf Technol 20(2):153-163

5. Brinksmeier E, Cammett JT, König W, Leskovar P, Peters J, Tönshoff HK (1982) Residual stresses - measurement and causes in machining processes. CIRP Ann Manuf Technol 31(2):491-510

6. Malkin S (1978) Burning limits for surface and cylindrical grinding of steels. Ann CIRP 27(1):233-236

7. Brinksmeier E (1991) Prozess- und Werkstückqualität in der Feinbearbeitung. VDI-Verlag, Düsseldorf. Fortschritt-Berichte VDI Reihe 2 Nr. 234

8. Heinzel C (2009) Schleifprozesse verstehen: Zum Stand der Modellbildung und Simulation sowie unterstützender experimenteller Methoden. Shaker, Aachen. Forschungsberichte aus der Stiftung Institut für Werkstofftechnik Bremen 47

9. Stenberg N, Proudian J (2013) Numerical modelling of turning to find residual stresses. Proced CIRP 8:258-264

10. Dehmani H, Salvatore F, Hamdi H (2013) Numerical study of residual stress induced by multi-steps orthogonal cutting. Proced CIRP 8:299-304

11. Arrazola PJ, Özel T, Umbrello D, Davies M, Jawahir IS (2013) Recent advances in modelling of metal machining processes. CIRP Ann Manuf Technol 62(2):695-718

12. Lazoglu I, Ulutan D, Alaca BE, Engin S, Kaftanoglu B (2008) An enhanced analytical model for residual stress prediction in machining. CIRP Ann Manuf Technol 57(1):81-84

13. Jawahir IS, Brinksmeier E, M'Saoubi R, Aspinwall DK, Outeiro JC, Meyer D, Umbrello D, Jayal AD (2011) Surface integrity in material removal processes: recent advances. CIRP Ann Manuf Technol 60(2):603-626

14. Brinksmeier E, Meyer D, Heinzel C, Lübben T, Sölter J, Langenhorst L, Frerichs F, Kämmler J, Kohls E, Kuschel S (2018) Process signatures - the missing link to predict surface integrity in machining. Proced CIRP 71:3-10

15. Meyer D, Kämmler J (2016) Surface integrity of AISI 4140 after deep rolling with varied external and internal loads. Proced CIRP 45:363-366

16. Langenhorst L, Borchers F, Heinzel C (2018) Analysis of internal material loads and resulting modifications for grinding with mechanical main impact. Proced CIRP (accepted for publication)

17. Tausendfreund A, Stöbener D, Dumstorff G, Sarma M, Heinzel C, Lang W, Goch G (2015) Systems for locally resolved measurements of physical loads in manufacturing processes. CIRP Ann Manuf Technol 64(1):495-498

18. Meyer H, Epp J, Zoch H-W (2016) In situ X-ray diffraction investigation of surface modifications in a deep rolling process under static condition. Mater Res Proc 2:431-436

19. Hertz H (1881) Über die Berührung fester elastischer Körper. Journal für die reine und angewandte Mathematik 92:156-171

20. Frerichs F, Lübben T (2018) Development of process signatures for manufacturing processes with thermal loads without and with hardening. Proced CIRP 71:418-423

21. Marek R, Nitsche K (2015) Praxis der Wärmeübertragung: Grundlagen; Anwendungen; Übungsaufgaben. 1st edn. Carl Hanser Fachbuchverlag

22. Klocke F, Schneider S, Mohammadnejad M, Hensgen L, Klink A (2017) Inverse simulation of heat source in electrical discharge machining (EDM). Proced CIRP 58:1-6

23. Klocke F, Mohammadnejad M, Zeis M, Klink A (2018) Investigation on the variability of existing models for simulation of local temperature field during a single discharge for electrical discharge machining (EDM). Proced CIRP 68:260-265

24. Klocke F, Schneider S, Ehle L, Meyer H, Hensgen L, Klink A (2016) Investigations on surface integrity of heat treated $42 \mathrm{CrMo} 4$ (AISI 4140) processed by sinking EDM. Proced CIRP 42:580-585

25. Klocke F, Mohammadnejad M, Hess R, Harst S, Klink A (2018) Phase field modeling of the microstructure evolution in a steel workpiece under high temperature gradients. Proced CIRP 71:99-104

26. Willert M, Riemer O, Brinksmeier E (2018) Surface integrity in precision turning of steel. Int $\mathrm{J}$ Adv Manuf Technol 94(1-4):763-771

27. Dumstorff G, Willert M, Riemer O, Lang W (2017) Characterizing precision cutting process by workpiece integrated printed thermocouples. In: Proceedings of the 17th international conference of the EUSPEN, p 59

28. Buchkremer S, Klocke F (2017) Modeling nanostructural surface modifications in metal cutting by an approach of thermodynamic irreversibility: derivation and experimental validation. Continuum Mech Thermodyn 29(1):271-289

29. Buchkremer S (2017) Irreversible thermodynamics of nanostructural surface modifications in metal cutting. 1st edn. Edition Wissenschaft Apprimus 2017, Band 8

30. Buchkremer S, Klocke F (2017) Compilation of a thermodynamics based process signature for the formation of residual surface stresses in metal cutting. Wear 376-377:1156-1163

31. Klocke F, Harst S, Zeis M, Klink A (2016) Energetic analysis of the anodic double layer during electrochemical machining of 42CrMo4 steel. Proced CIRP 42:396-401

32. Klocke F, Harst S, Ehle L, Zeis M, Klink A (2018) Surface integrity in electrochemical machining processes: an analysis on material modifications occurring during electrochemical machining. Proc Inst Mech Eng Part B J Eng Manuf 232(4):578-585

33. Mehrafsun S, Harst S, Hauser O, Eckert S, Klink A, Klocke F, Vollertsen F (2016) Energy-based analysis of material dissolution behavior for laser-chemical and electrochemical machining. Proced CIRP 45:347-350

34. Klocke F, Harst S, Karges F, Zeis M, Klink A (2017) Modeling of the electrochemical dissolution process for a two-phase material in a passivating electrolyte system. Proced CIRP 58:169-174

35. Klocke F, Harst S, Zeis M, Klink A (2018) Modeling and simulation of the microstructure evolution of $42 \mathrm{CrMo} 4$ steel during electrochemical machining. Proced CIRP 68:505-510

36. Klink A (2016) Process signatures of EDM and ECM processes - overview from part functionality and surface modification point of view. Proced CIRP 42:240-245

37. Kochmann J, Rezaeimianroodi J, Reese S, Svendsen B (2014) Two-dimensional elastic phase-field simulation of fcc to bcc martensitic phase transformations in polycrystals. Proc Appl Math Mech 14(1):397-398

38. Yoshimoto C, Takaki T (2014) Multiscale hot-working simulations using multi-phase-field and finite element dynamic recrystallization mode. Iron Steel Inst Jpn 54:452-459

39. Rezaei S, Kochmann J, Wulfinghoff S, Reese S (2017) Cohesize zone-based modeling of nano-coating layers for the purpose of establishing process signatures. GAMM Mitteilungen 40(1):71-88

40. Miehe C, Schotte J, Schröder J (1999) Computational micromacro transitions and overall moduli in the analysis of polycrystals at large strains. Comput Mater Sci 16:372-382

41. Smit RJM, Brekelmans WAM, Meijer HEH (1998) Prediction of the mechanical behavior of nonlinear heterogeneous systems by multi-level finite element modeling. Comput Methods Appl Mech Eng 155:181-192 
42. Hashin Z, Shtrikman H (1983) On some variational principles in anisotropic and nonhomogeneous elasticity. J Appl Mech 36:481-505

43. Hill R (1963) Elastic properties of reinforced solids: some theoretical principles. J Mech Phys Solids 11:357-372

44. Spahn J, Andrae H, Kabel M, Müller R (2014) A multiscale approach for modeling progressive damage of composite materials using fast Fourier transforms. Comput Methods Appl Mech Eng 268:871-883

45. Kochmann J, Mianroodi JR, Wulfinghoff S, Svendsen B, Reese S (2016) Two-scale, FE-FFT- and phase-field based computational modeling of bulk microstructure evolution and macroscopic material behavior. Comput Methods Appl Mech Eng 305:89-110

46. Kochmann J, Wulfinghoff S, Ehle L, Mayer J, Svendsen B, Reese S (2018) Efficient and accurate two-scale FE-FFT-based prediction of the effective material behavior of elasto-viscoplastic polycrystals. Comput Mech 61(6):751-764

47. Suquet $P$ (1997) Continuum micromechanics. CISM International Center for Mechanical Sciences, vol 377. Springer, Vienna

48. Prakash A, Lebensohn A (2009) Simulations of micromechanical behavior of polycrystals: finite element versus fast Fourier transforms. Modell Simul Mater Sci Eng 17:064010

49. Kochmann J, Brepols T, Wulfinghoff S, Svendsen B, Reese S (2018) On the computation of the exact overall consistent tangent moduli for non-linear finite strain homogenization problems using six finite perturbations. In: 6th European conference on computational mechanics (ECCM 6)

50. Chen LQ (2002) Phase-field models for microstructure evolution. Annu Rev Mater Sci 32:113-140

51. Ostoja-Starzewski M (2002) Towards stochastic continuum thermodynamics. J Non-Equilib Thermodyn 27:335-348

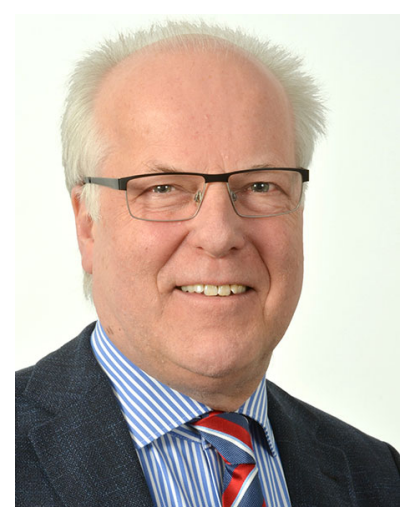

E. Brinksmeier is Full Professor at the University of Bremen and Deputy Managing Director of the Leibniz Institute for Materials Engineering IWT (former Institute of Materials Science IWT) in Bremen, Germany. After receiving his Dr.-Ing. Degree in 1982 from the University of Hannover he worked as Chief Engineer at the Institute for Production Engineering and Machine Tools (IFW). He finished his habilitation in 1992 and became a Full Professor for manufacturing technologies at the University of Bremen. From 1992 to 2018 he was director of the Foundation Institute of Materials Science (IWT) and founder and director of the Laboratory for Precision Machining (LFM). His scientific interests and research areas lie in the field of advanced manufacturing processes with special focus in the areas of grinding, ultraprecision machining, development of sensor-integrated tools, development of advanced metalworking fluids, and the generation of functional surfaces by machining. Amongst others he is Honorary Fellow and Past President of the International Academy for Production Engineering (CIRP). He is coordinator of the Collaborative Research Center on "Process Signatures" with participation of the universities of Bremen, Aachen (Germany) and Oklahoma State University at Stillwater (USA).

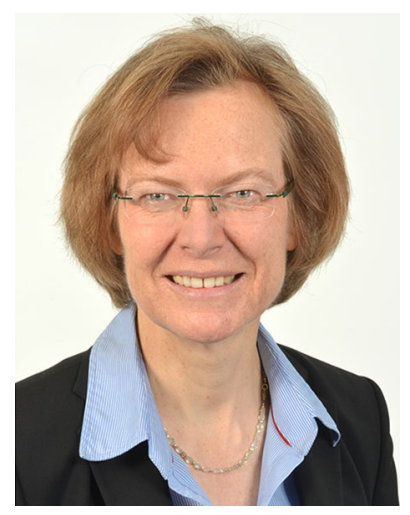

S. Reese is Full Professor at RWTH Aachen University and Director of the Institute of Applied Mechanics (IFAM) in Aachen, Germany. After receiving her Dr.-Ing. Degree in 1994 from the TU Darmstadt she worked as Assistant Professor at TU Darmstadt and University of Hannover. She finished her habilitation for Mechanics in 2000 at the University of Hannover. Between 2000 and 2005 she was Associate Professor at the Ruhr University Bochum and from 2005 until 2009 she was Full Professor at the TU Braunschweig. Since November 2009 she is Full Professor at the RWTH Aachen University. Her scientific interests and research areas focus on the modelling of complex materials as well as on the efficient numerical simulation, e.g. in the areas of finite element technology, model reduction and multi-scale modelling. The experimental branch of research concentrates on the multiaxial mechanical behavior of soft materials (e.g. biomaterials) but also metals. Amongst others she is Fellow of the International Association for Computational Mechanics (IACM) and Treasurer of the European Mechanics Society (Euromech). She is co-coordinator of the Collaborative Research Center on "Process Signatures".

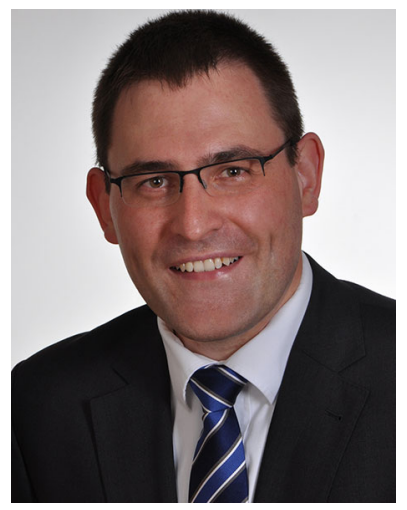

A. Klink is Chief Engineer for the Department of Electro Physical and Chemical Processes at the Chair of Manufacturing Technology of the Laboratory for Machine Tools and Production Engineering (WZL) of RWTH Aachen University. He received his Dr.Ing. Degree in 2009 from the RWTH Aachen University. His scientific interests and research areas especially lie in the field of advanced manufacturing processes like Electrical Discharge Machining (EDM) and Electrochemical Machining (ECM). $\mathrm{He}$ is member of the Collaborative Research Center on "Process Signatures" and leads the research team "Chemical and ThermoChemical Processes".

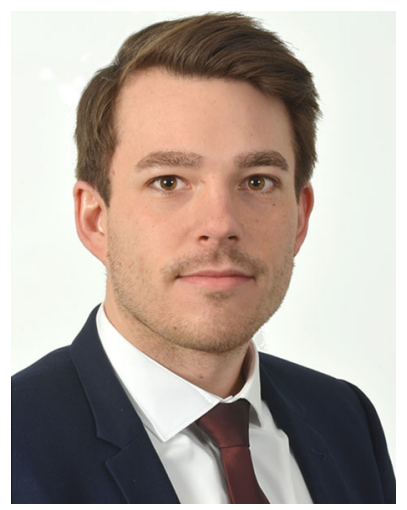

L. Langenhorst received his M. Sc. degree in Production Engineering at the University of Bremen, Germany. Since 2016 he works as a Research Engineer at the Leibniz Institute for Materials Engineering IWT. His research focuses on modeling and simulation of advanced manufacturing processes. In the Collaborative Research Center on "Process Signatures" he currently develops process models for grinding and milling to gain a deeper insight into the mechanisms leading to changes in the surface integrity. 


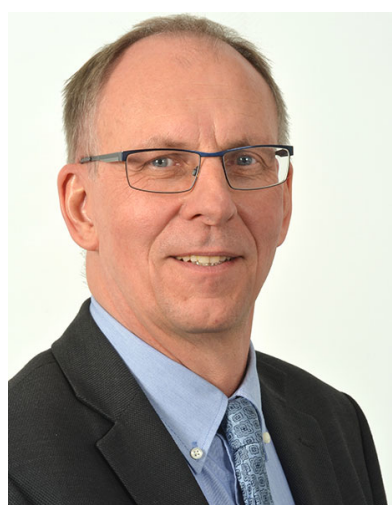

T. Lübben studied physics at the University of Bremen, Germany. He defended his Ph.D. thesis in 1994. Since 1986 he works at the Leibniz Institute for Materials Engineering IWT. From 2001 to 2011 he was the managing director of the Collaborative Research Center "Distortion Engineering" which was established by the German Research Foundation DFG at the University of Bremen in cooperation with IWT Bremen. His research interests are distortion in general and especially quenching technology with focus on the measurement of heat transfer in gases and fluids, gas quenching, press quenching, and simulation of quenching processes (phase transformation and distortion). He is member of the Collaborative Research Center on "Process Signatures" and leads the research team "Thermal Continuous Processes".

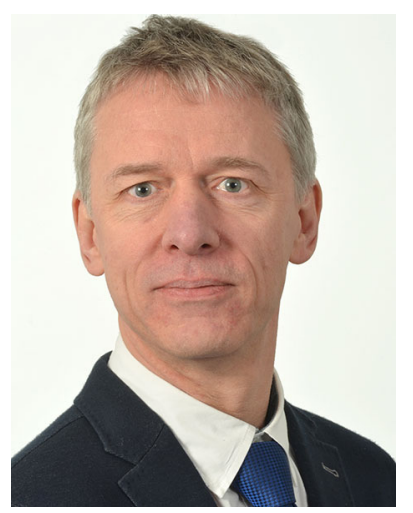

M. Meinke received his doctoral degree in 1993 from RWTH Aachen University. Since then he is the head of the CFD department at the Institute of Aerodynamics of RWTH Aachen University and teaches Compuational Fluid Dynamics to Bachelor and Master Students. His research focus lies on the numerical simulation of phenomena related to fluid mechanics and aeroacoustics. He has published more than 80 conference papers and 140 journal articles. He is member of the Collaborative Research Center on "Process Signatures" and leads the research team "Thermal Discontinuous Processes".

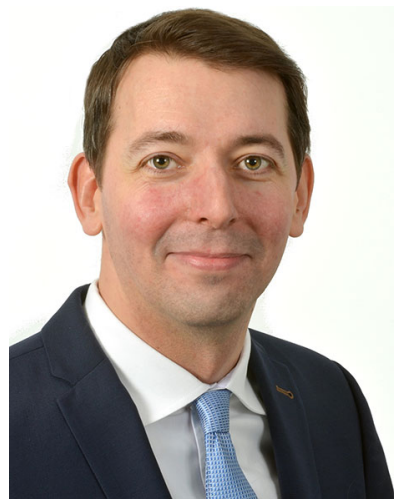

D. Meyer is the Chief Engineer of the Division Manufacturing Technologies at the University of Bremen and the Leibniz Institute for Materials Engineering IWT and the Head of a Junior Research Group focusing on the functionalization of metal surfaces by manufacturing processes at the IWT. He received his Dr.-Ing. Degree in 2012 from the University of Bremen. His research focuses on the complex interrelations between manufacturing processes and the surface integrity of metallic parts. As a studied biologists, he also considers highly interdisciplinary effects, e.g. in metalworking-fluidrelated research. He has been awarded the F.W. Taylor Medal by the International Academy for Production Engineering CIRP, the DFG Heinz Maier-Leibnitz-Prize by the German Research Foundation DFG, and the Otto Kienzle Commemorative Coin by the German Academic Society for Production Engineering WGP. He is Associate Member of member of the International Academy for Production Engineering CIRP and of the Collaborative Research Center on "Process Signatures" and leads the research team "Mechanical Processes" of the center.

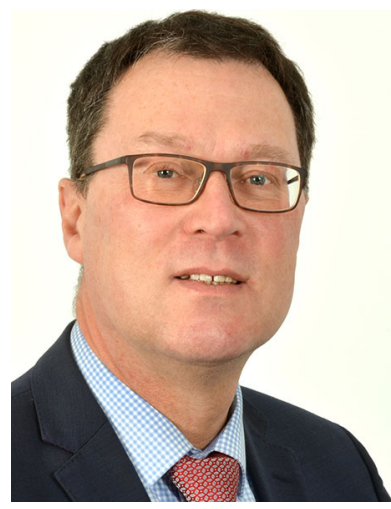

0. Riemer is head of the Laboratory for Precision Machining (LFM) at the Leibniz Institute for Materials Engineering IWT in Bremen, Germany. He graduated in 1992 in Mechanical Engineering from Technical University of Braunschweig and received his Dr.-Ing. Degree in 2001 from the University of Bremen. His scientific interests and research areas lie in the field of advanced manufacturing processes with special focus in the areas of ultraprecision machining of optics like diamond machining, precision grinding and polishing and surface as well as materials characterization. Amongst others Dr. Riemer is Council member of the European Society for Precision Engineering and Nanotechnology (euspen). He is member of the Collaborative Research Center on "Process Signatures" and leads the research team "Thermo-Mechanical Processes".

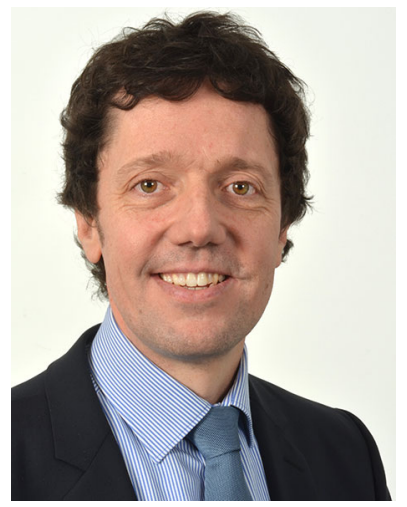

J. Sölter studied physics at the University of Oldenburg, Germany, the University of Colorado Denver, USA, and the University of Freiburg, Germany and works as a Postdoc at the University of Bremen and at the Leibniz Institute for Materials Engineering IWT in Bremen, Germany. He received his Dr.-Ing. Degree in 2010 from the University of Bremen. His scientific interests and research areas lie in the field of modeling and simulation of advanced manufacturing processes and their impact on surface integrity and distortion. He participated in the Research Affiliate Program of the International Academy for Production Engineering CIRP and is member of the MAPEX Center for Materials and Processes at the University of Bremen. Since 2014 he is the managing director of the Collaborative Research Center on "Process Signatures". 